\title{
Chile Tree Ring Chronologies as Proxy Data for Solar Variability
}

\author{
Nivaor Rodolfo Rigozo $(1,2)$, Alan Prestes $(2)$, \\ Daniel Jean Roger Nordemann ${ }^{(2)}$, Ezequiel Echer ${ }^{(2)}$, \\ Luís Eduardo Antunes Vieira(2) and \\ Heloisa Helena de Faria $(2)$ \\ ${ }^{1}$ Faculdade de Tecnologia Thereza Porto Marques - FAETEC, \\ CEP 12308-320, Jacareí, Brazil
}

Fone: 551239524231

${ }^{2}$ Instituto Nacional de Pesquisas Espaciais - INPE,

CP 515, 12201-970 São José dos Campos, Brazil.

Fone: 551239456840 - Fax 551239456810

E-MAIL: rodolfo@dge.inpe.br, prestes@dge.inpe.br, nordeman@dge.inpe.br, eecher@dge.inpe.br, eduardo@dge.inpe.br,hfarai@dge.inpe.br

\begin{abstract}
Tree ring index chronologies, representing standardized annual growth rates for Fitzroya cupressoides at Cordillera de la Costa de Osorno in Chile, have been employed for the search of solar periodicities during the last 400 years. Spectral analysis of tree ring series by multitaper method has determined significant periodicities at about 21 and 10.7 years. These values are close to two known present basic solar activity periods at 22 and 11 years (Hale and Schwabe cycles). Other periodic component appears at 5 years, which may also be related to solar variations. The short periods found probably may be due the environmental and climatic influences. The application of band pass filter techniques shows that the 11 year cycle present in tree ring series correlates with the sunspot numbers with a time lag of about two years, since AD 1700, the extent of accurate sunspot record interval.
\end{abstract}

Keywords: Tree Ring Index - Solar Variability - Multitaper Spectral Analysis - Climatic Change

\section{Introduction}

Dramatic variations such as sunspots numbers have been observed for more than a century in solar phenomena. Unambiguous confirmation of real variations in the Sun's radiation at the Earth is, however, relatively recent; only during the past decade have satellite measurements revealed the variability of the solar constant. During last centuries, maxima and minima in solar activity have occurred approximately every 11 years. This is demonstrated clearly by the sunspot data. The Sun also exhibits variability over time scales both longer and shorter than its dominant 11-year cycle (Hudson, 1988; Foukal, 1990). Techniques using cosmogenic isotopes have reconstructed solar activity variations on longer time scales. Two isotopes are commonly used, carbon-14 and beryllium-10, both produced by cosmic rays. Galactic cosmic rays are modulated by changes in the strength of the interplanetary magnetic field arising from changes 
in solar activity (Hoyt and Schatten, 1997). The existence of century scale variations caused by solar activity has been confirmed from ${ }^{14} \mathrm{C}$ dating (Stuiver and Quay, 1980) and ${ }^{10} \mathrm{Be}$ ice-core data (Beer et al., 1988). The sun's long-term behaviour also shows transient dynamics such as the Maunder minimum from AD 1645 to 1715 (Eddy, 1976), characterized by a striking decrease of solar activity.

Recent techniques have been using trees ring as a possible proxy of the solar activity variations in the past, but it was not still possible to observe clearly a correspondence between tree rings and the solar activity, due to overlap of the climatic signs masking the signs of solar origin. Mori (1981) also found strong evidence for a 11-year solar cycle signal in a tree ring series from Formosa. Cross spectral analysis with solar data demonstrated a high coherence level at a frequency near 11-years with time lag of about 2.8 years. More recently Murphy and Veblen (1992) have reported a dominant cycle of 11-years existing in tree ring index from Colorado. Although the casual connection between solar variations and growth-rings is more complex than that between solar variations and cosmogenic isotopes, several studies suggest that such connections may exist (Kurths et al., 1993). This led us to investigate ring-width records of solar periodicities of a coniferous tree with an age of 419 years by spectral analysis using multitaper method.

\section{Tree Ring Data}

The tree ring width data set used in this investigation has been obtained by Tree Ring Image Interactive Treatment (TRIIT) (Rigozo, 1998). Only one disk sample of Fitzroya cupressoides native species was studied and presents a 419 year chronology from 1575 to 1993 AD, as determined by the Department of Botany, University of Chile according to standard dendrochronological procedures for the chosen radius series. This chronology has been used to investigate the possibility that solar activity induces periodical signals in growth rings at Cordillera de la Costa de Osorno in Chile (Lat: 41 $00^{\prime} \mathrm{S}$; Long: $73^{\circ} 50^{\prime} \mathrm{W}$; Alt: 1000m). The tree ring series has been standardized by a twopeak gaussian function. The tree ring index time series is illustrated in Figure 1 along with an indication of the period of Maunder-minimum of low solar activity.

\section{Spectral Analysis and Results}

The spectral estimate is a powerful method to analyze data and it is frequently used to study geophysical processes. Many techniques have been developed to analyze long stationary time series. However, these techniques are not universally applied for time series that involve non-stationary processes of short duration. The main problem has been to isolate spectral frequencies amid a noise, to obtain a spectrum estimation with a low spectral leakage and minimum variance (Stearns and Ruth, 1993). A new technique of spectral estimate, known as spectral analysis by multitaper, firstly presented by Thomson (1982), has been applied in the analysis of time series, mainly in seismology (Park et al., 1987) and climatology (Thomson, 1990) areas. In this method, the data are multiplied by a window function, as it happens in the conventional methods, but for several resistant functions to spectral leakage. By this, several spectral estimates are obtained from a time series, that can be combined to form only one spectral estimate. The routine used in this work to determine the spectral estimate by multi-tapers was improved from former ones and used by Vieira (1998).

After removal of the long range tendencies, as above described, from tree ring time series, the spectral analysis is performed. Figure 2 shows a statistical estimate of significance of the frequency registered in the growth rings, in which the spectral probability has been calculated using the multitaper method. The significant peaks at both lower and high frequencies suggests that this species, growing at high altitudes sites, is very responsive to longer and shorter periods and to climatic factors influencing growth patterns. 
Figure 2 show periods as high as $140.0 \mathrm{yr}$ with an estimate of the statistical significance of $90 \%, 38.0 \mathrm{yr}$ with an estimate of $95.2 \%, 20.9 \mathrm{yr}$ with an estimate of $95 \%, 17.5 \mathrm{yr}$ with an estimate of $95.2 \%, 13.1 \mathrm{yr}$ with an estimate of $93 \%$ and $10.7 \mathrm{yr}$ with an estimate of $97 \%$. It may be observed that there exist two peaks, with a high statistical estimate, close to the 11 years, 13.1 and 10.7 years. The Figure 2 shows high statistical estimates for the short periods, this represents a strong response of the tree rings to environmental influences. Some of these observed periods present estimated statistical significance above $95 \%$, as it is the case of $6.3,4.3,4.0,2.7,2.6,2.3$ and 2.03 years.

Assuming that these signals have a solar origin, it would appear that the length of the solar cycle over this period was near 10.7 years and less than the current average of 11.1 years obtained by Murphy (1990). The same result was observed also in tree rings by Murphy and Palmer (1992), who found between 426 years (close to AD 177), a mean period of 10.5 years for the length of the "11-year" solar cycle. An equivalent result was observed by these authors, for the "22-year" solar cycle, who obtained for the length of this solar cycle in the tree ring index, for the same time interval, a value of 20.9 years.

The cross correlation between the two time series, tree ring index and relative sunspot number (NOAA, 1994), can demonstrate the periodic equivalence of two time series and establish their phase relationship along with an indication of the common interval over which the periodicity has persisted. Figure 3 which gives the magnitude of the correlation coefficient at various time lags illustrates this situation. Here at lag -2 the value of cross correlation is approximately 0.18 determined a positive correlation clearly exists with a growth response time lag of about 2 years in relation to solar activity phase.

The attention has been directed towards identifying epochs of very low solar activity, based on information derived from indirect sources. One of these epochs is it Maunder minimum from AD 1645 to 1715 (Eddy, 1976). Figure 4 present the spectral analysis for three periods, (A) before Maunder from AD 1575-1645, (B) during Maunder from AD 1645-1715 and (C) after Maunder from AD 1715-1993.

In Figure 4(A) only a long period, 35.7 years, has been found with an estimated probability above 95\%, and three short periods, 5.1, 3.2 and approximately 2.5 years, have been fond with an estimated probability above $93 \%$. In Figure 4(B) only short periods show a high estimated statistical significance, 3.93 .1 and 2.1 years, above 95\%. The interesting fact is a period found close to the 11-year solar cycle but with a low estimate of probability, which turns that result not reliable. Figure 4(C) shows periods due to the solar cycles of 22 and 11-years with an estimated probability of $99 \%$ for 21.3 years, $91 \%$ for 13.3 years and $94 \%$ for 9.3 years. It was also found a high estimated statistical significance of $94 \%$ for periods between 93 and 140 years. Short periods were also found with an estimated statistical significance above 93\%, 6.3, 4.4, 3.5, 3.2 and 2.3 years. This represents that the signals of solar origin are dominant after Maunder minimum.

\section{Discussion and Conclusions}

The long-standing suggestion that variations in the Sun's total irradiance (the "solar constant") on time scales of decades to millennia may have been the cause of long-term climate variations has been revived in recent years by spacecraft radiometric measurements (Wilson and Hudson, 1991) showing that the Sun's total irradiance has varied more or less in phase with solar magnetic activity as measured by the sunspot number since peak of solar cycle 21 in 1980. As was originally pointed out by Eddy (1976), the Maunder minimum coincided roughly with one the coldest episodes of the Little Ice Age, when global temperatures have been estimated to have been 1 to $1.5^{\circ} \mathrm{C}$ colder than modern temperatures (Crowley and North, 1991; Bradley and Jones, 1993). Thus, it may be thought that variations in 
the total irradiation from the Sun can induce variations in tree growth by some mechanisms which involve sun induced global and regional climatic variations and specific sensitivity of trees to ambient variations which intervene as limiting factors when in stress conditions.

Confirmation of ring width variations may be attributed, at least in part, to anomalous solar activity. Many early spectral studies failed to find any significant evidence of solar periodicities (La Marche and Fritts, 1972). However, recent spectral studies have shown strong evidences of a 11-year period in tree ring data. Murphy (1990) found evidences of a 11-year solar signal in tree ring data from Australia, with a time lag of 3 years. He also found periods close to the 22-year and 90 year (Gleissberg cycle) solar cycles. We also found a period close to the 11 year-old solar cycle, but with a time lag of 2 year for the sample of Chile, and a strong evidence of the signal of the 22-year cycle. These same results were found by Kurths et al. (1993) in samples of trees of Miocene from Germany, where they observed periods of 12.5 years and the second harmonic of 11 year cycle in 5.5 years. We observed the second harmonic at 5.1 years for the period before Maunder minimum. For the period after the Maunder minimum, it was found at 4.8 years but this periodicity is not clearly related to a solar signal..

Evidence shows that during the Maunder minimum from 1645 to 1715 , the climate was anomalous. Studies of tree rings in the American Southwest suggested that the late 1600s was a period of drought although the Scandinavian tree rings shows the Maunder minimum was a period of relative warmth (Douglas, 1936). The same author also wrote that during the Maunder minimum the 11-year tree ring cycle disappeared. All these anecdotal and regional accounts give a confusing and contradictory picture of the Maunder minimum climate. Rind and Overpeck (1993) used the Goddard Institute for Space Studies General Circulation Model (GISS/GCM) to model the consequences of prolonged decrease in solar irradiance such as which may have occurred during the Maunder minimum They found that while most of the world cooled, certain regions were actually warmer. This simulation revealed warmer regions east of Scandinavia where the tree rings data shows a warmer climate. This model also evidenced a cold period in South America and particularly in Chile, where we found no significant statistical evidence for the 11-year solar cycle in the tree ring series from Chile, during the Maunder minimum, but this negative result does not allow to conclude that chronology intervals without 11-year solar cycle correspond systematically to cold weather periods.

The spectral analysis of tree ring data from Chile presented strong evidences for the 11-year solar cycle, with a time lag about 2 years, and the cycle of Hale and these are dominant after Maunder minimum, 1715-1993 AD. We observed also the second harmonic at 5.1 years for the period before Maunder minimum. The Gleissberg cycle presents a periodicity of 93 to 140 years, for the post-Maunder minimum period. The short periods observed can be due the environmental influences and climatic. The results found are in agreement with other record studies of the solar activity in the past, such as the concentrations of the cosmogenic isotopes carbon-14 (Stuiver and Quay, 1980) and beryllium10 (Beer et al., 1988). More studies of regional tree ring data, as this, become necessary to determine the relative importance of the solar influences in the Sun-Earth system, in the past, since tree rings are seemingly becoming an excellent proxy data base for solar variability.

\section{Acknowledgments}

The authors wish to thank Conselho Nacional de Desenvolvimento Científico e Tecnológico - CNPq for support granted to this research (Project APQ 474185/2003-6).

\section{References}


Beer, J., Siegenthaler, U., Bonani, G., Finkel, R. C., Oeschger, H., Sater, M., \& Woelffi, W., 1988, Nature, 331, 675679.

Bradley, R. S., \& Jones, P. D., 1993, The Holocene, 3, 367-376.

Corwley, T. J., \& Noth, G. R., 1991, Oxford University Press, New York.

Douglas, A. E., 1936, Climatic cycles and tree growth: A study of cycles. Washington, DC. Carnegie Institute of Washington Publications, vol.3.

Eddy., J. A., 1976, Science, 192, 1189-1202.

Foukal, P., 1990, Solar Astrophysics, John Wiley, New York.

Hoyt, D. V., \& Schatten, K. H., 1997, Oxford University Press, New York.

Hudson, H. S., 1988, Annu. Rev. Astron. Astrophys., 26, 473-507.

Kurths, J., Spiering, Ch., Müller-Stoll, W., \& Striegler, U., 1993, Terra Nova, 5, 359-363.

La Marche, V. C., \& Fritts, H. C., 1972, Tree-Ring Bulletin., 32, 19-33.

Mori, Y., 1981, Journal of Climatology, 1, 345-353.

Murphy, J. O. 1990, Proceedings ASA, 8, 292-297.

Murphy, J. O., \& Palmer, J. G., 1992, Proc. Astron. Soc. Aust., 10, 68-70.

Murphy, J. O., \& Veblen, T. T., 1992, Proc. Astron. Soc. Aust., 10, 64-67.

NOAA, 1994, National Geophysical Data Center, http://www.ngdc.noaa.gov/stp/stp.html.

Park, J., Lindberg, C. R., and Thomson, D. J., 1987, Geophysical Journal Royal Astronomical Society, 91, $755-794$.

Rigozo, N. R., 1998 , Instituto Nacional de Pesquisas Espaciais, São Paulo.

Rind, D., \& Overpeck, J. T., 1993, Quaternary Science Reviews, 12, 357-374.

Stearns, S. D., \& Ruth, A. D., 1993, Prentice Hall Signal Processing Series, New Jersey.

Stuiver, M., \& Quay, P. D., 1980, Science, 207, 11-19.

Thomson, D. J., 1982, IEEE Proc., 70, 1055-1096.

Thomson, D. J., 1990, Phil. Trans. R. Soc. Lond., 33, 539-597.

Vieria, L. E. A., 1998, Master Degree, Instituto Nacional de Pesquisas Espaciais, São José dos Campos, Brazil. Willson, R. C., \& Hudson, H. S., 1991, Nature, 351, 42-44.

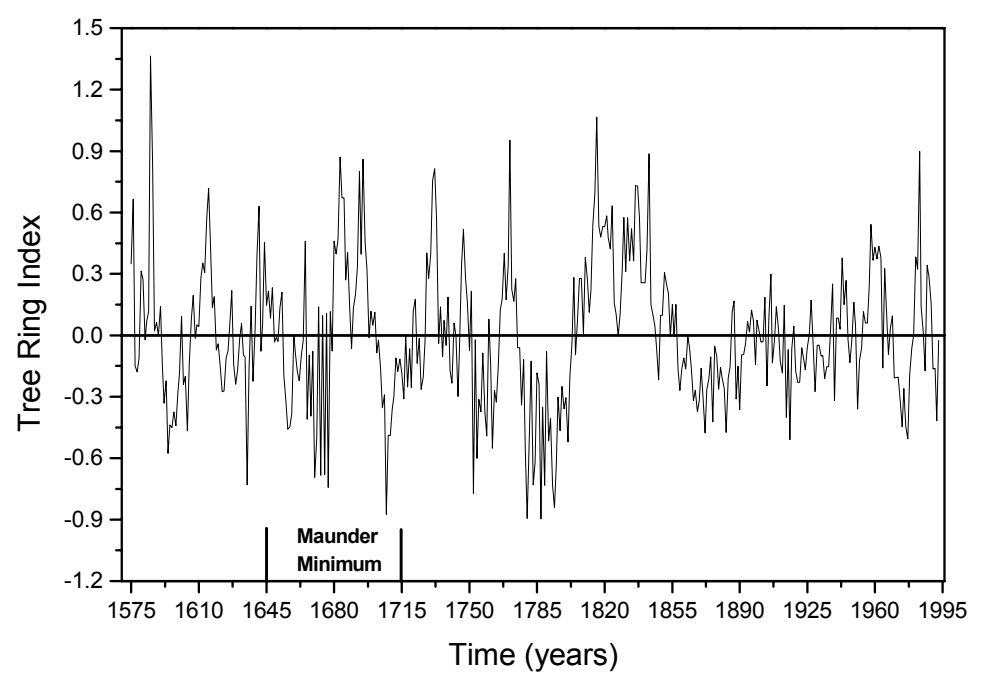

Figure 1 - Tree ring index time series extending from 1575 to 1993 AD with zero mean. The Maunder minimum period (1645 to 1715 AD) of very low solar activity, as determined by carbon-14 dating, has been indicated. 


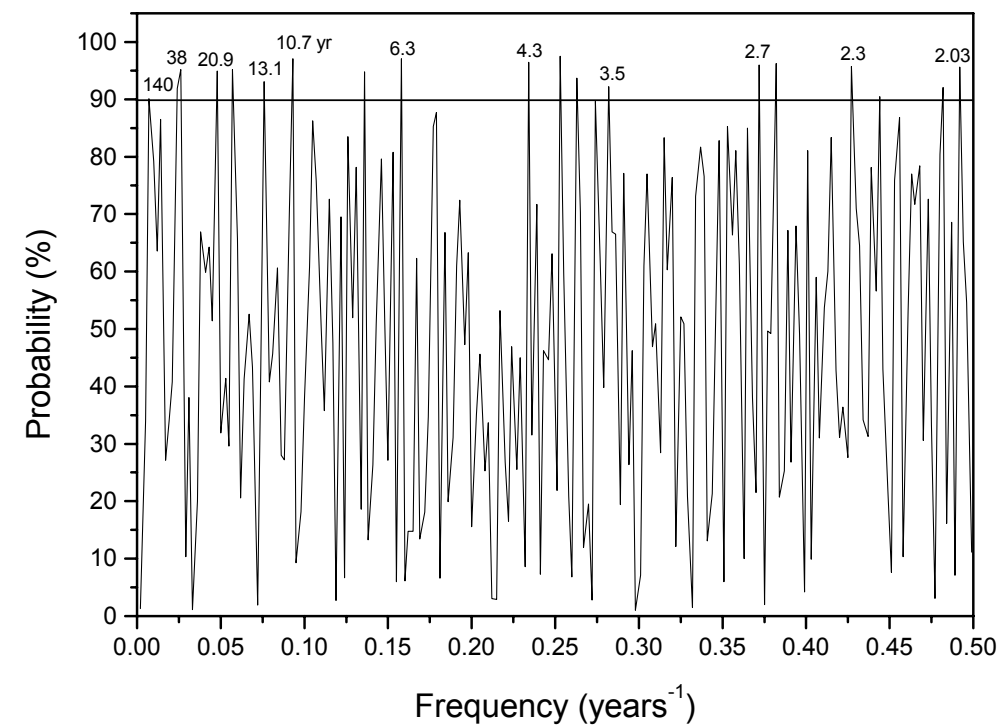

Figure 2 - Spectral probability at tree ring index time series over AD 1575-1993.

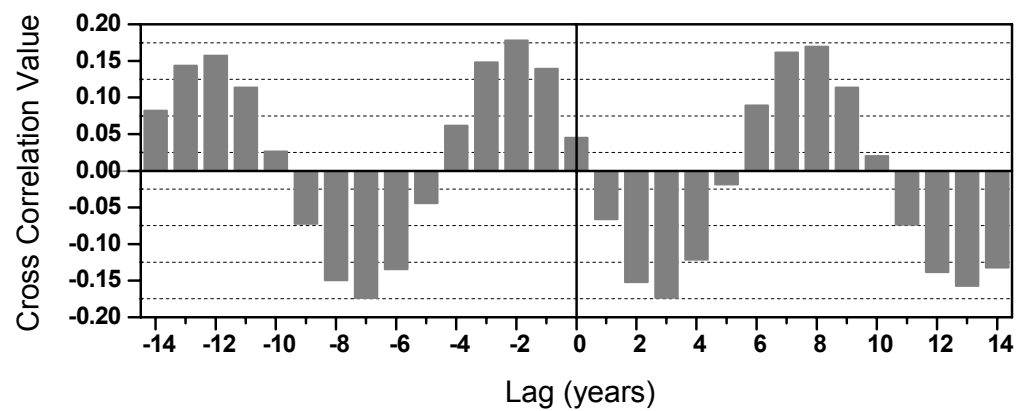

Figure 3 - Cross correlation of tree ring index and sunspot numbers over the common period of AD 1700 to 1993.
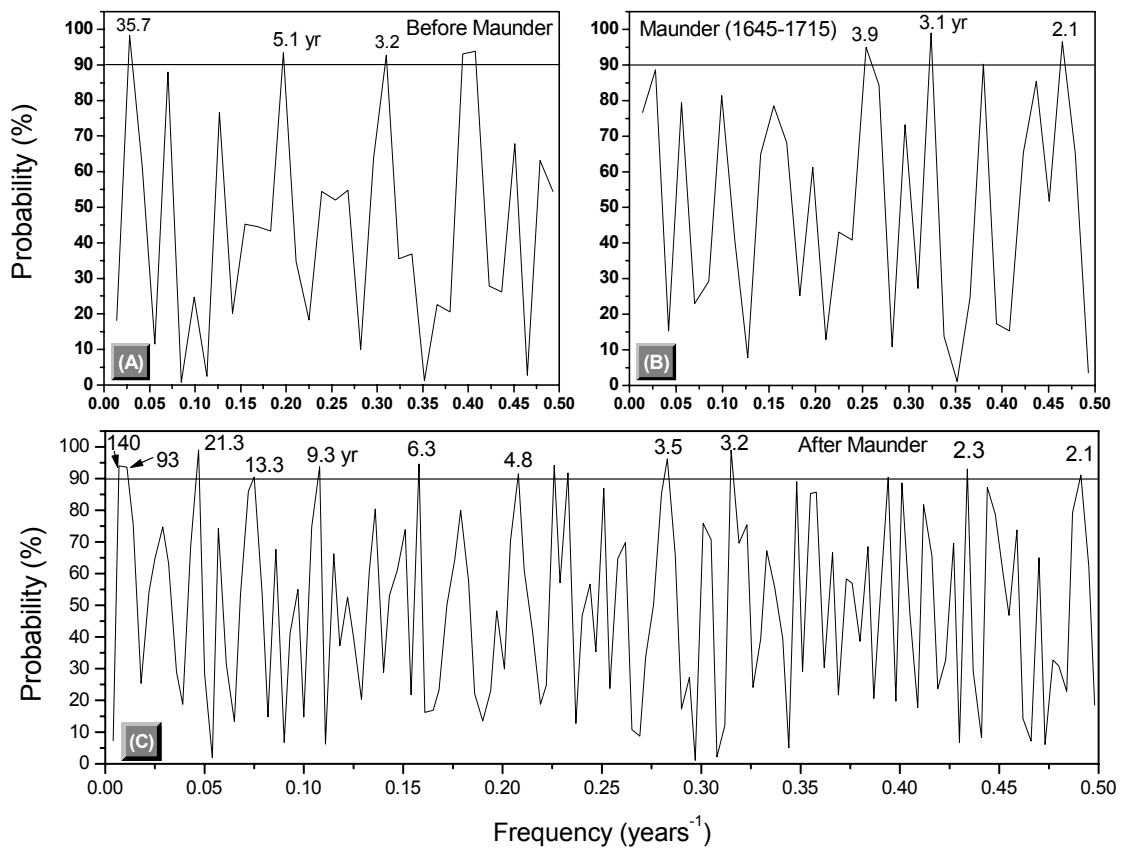

Figure 4 - Spectral probability obtained at (A) before the time of Maunder minimum AD 1575-1645, (B) during the time of Maunder minimum AD 1645-1715 and (C) after the period of the Maunder minimum AD 1715-1993. 